\title{
UV inscribed long period gratings with femtosecond ablated axial fibre slots for polarization control
}

\author{
Edward Davies ${ }^{1}$, Kyriacos Kalli $^{2 *}$, Charalambos Koutsides ${ }^{2}$. Tom Allsop ${ }^{1}$ and Lin Zhang ${ }^{1}$ \\ ${ }^{1}$ Photonics research group, Aston University, Birmingham, B47ET, United Kingdom \\ ${ }^{2}$ Nanophotonics Research Laboratory, Cyprus University of Technology, 3036, Lemessos, Cyprus
}

\begin{abstract}
We present data on the development a new type of optical fibre polariser and the characterisation of its wavelength properties. The device is fashioned using a two step process. Firstly, a standard UV long period grating (LPG) with a period of $330 \mu \mathrm{m}$ is inscribed into hydrogenated SMF-28, followed by femtosecond laser ablation of a groove parallel to the fibre axis. The UV inscribed LPGs have inherently low birefringence. However, the removal of the cladding layer parallel to the location of the LPG within the fibre core (as a result the ablation) modifies the cladding modes that couple with the LPG. Furthermore, the groove breaks the fibre symmetry introducing a non-uniform stress profile across the fibre cross section leading to significant birefringence. We show that increasing the depth of the groove increases the birefringence, and this behaviour coupled with the ability to control the wavelength location of the LPGs attenuations peaks results in a polariser able to operate at almost any wavelength and birefringence. The maximum birefringence reported here as polarisation mode splitting was approximately $39 \pm 0.1 \mathrm{~nm}$ with a polarisation loss of $10 \mathrm{~dB}$.
\end{abstract}

Keywords: Femtosecond laser, Long period grating, Polariser

\section{INTRODUCTION}

In line optical fibre polarisers are more favourable than their bulk optics counterparts because of their light weight, low insertion loss and high coupling efficiency to existing optical networks. Currently, there are three main approaches for polarisation control in optical fibres; i) side polished D-shaped optical fibre coated with a thin metallic layer; the core mode of the optical fibre selectively couples to the polarisation dependent surface plasmon mode ${ }^{1}$; ii) D-shaped optical fibre embedded in birefringent crystals, where the core mode interacts with the polarisation dependent crystals allowing for the extinction of one polarisation mode and the propagation of the orthogonal state ${ }^{2}$; iii) the UV laser inscription of Bragg grating structures tilted at $45^{\circ}$ from the fibre core axis; the tilt strongly out couples the $\mathrm{S}$ polarised light to the radiation modes, whilst the $\mathrm{P}$ polarised light remains bound in the fibre core ${ }^{3}$. These techniques are efficient but have disadvantages, such as the weakness of the D-shaped fibre and the consistency and repeatability of the inscription of the tilted grating structures in hydrogen loaded fibre. Here we report an in-line polariser than can theoretically work at any and multiple wavelengths since it is based on a modified long period grating (LPG). The LPG couples light from the core mode to the co-propagating cladding modes and when inscribed using a uniform refractive index change, as with UV laser inscription, they have inherently low birefringence. However, birefringence can be selectively introduced with a femtosecond laser-ablated groove "carved" into the fibre cladding. The ablation only occurs at the surface and the core mode remains unaffected, while the introduction of asymmetry creates birefringence for the cladding modes that is controlled by the ablated groove depth. This is characterised by a splitting in the LPG attenuation peak.

\section{UV AND FEMTOSECOND INSCRIPTION METHOD AND POLARISATION ANALYSIS}

The LPGs were inscribed into SMF-28, hydrogen-loaded optical fibre, using a frequency doubled argon ion laser (operating wavelength $244 \mathrm{~nm}$ ) for which a series of near identical LPGs were inscribed with a period of $330 \mu \mathrm{m}$. This period was chosen as it produced a strong attenuation peak at approximately $1550 \mathrm{~nm}$, offering two advantages.

*kkalli@cytanet.com.cy

21st International Conference on Optical Fiber Sensors, edited by Wojtek J. Bock, Jacques Albert, Xiaoyi Bao, Proc. of SPIE Vol. 7753, 77539G · C 2011 SPIE · CCC code: 0277-786X/11/\$18 · doi: 10.1117/12.885990 
Firstly, the device can be designed to operate for the $1550 \mathrm{~nm}$ optical communications window, and secondly, this attenuation peak is coincident with the centre of the broadband light source used for device characterisation and allows for large wavelength monitoring range. The LPGs were annealed at $100^{\circ} \mathrm{C}$ for 48 hours to stabilise the transmission spectrum. A femtosecond laser system with a $100 \mathrm{kHz}$ repetition rate, emitting $300 \mathrm{fs}$ pulses of energy $10 \mu \mathrm{J}$ at a wavelength of $1035 \mathrm{~nm}$, was used to micro-machine a slot into the fibre cladding above the region of the grating. A long working distance, high NA Mitutoyo lens was used to generate a small and highly accurate focal spot for the ablation of the micro-slot structures, which is critical to the direct write nature of the work. The fibre was suspended on fibre mounts on a high precision Aerotech air bearing 2D translation stage, for high accuracy motion of the fibre. The slot was made by multiple scans starting from the cladding edge then moving inwards towards the core. In this way, the ablated slot depth was controlled and repeatable. The slot lengths were made to be between $20-30 \mathrm{~mm}$ long and were visually inspected using a microscope to establish their size and shape. The polarization response was then investigated using a light source coupled to a polarization controller ( $<1$ degree polarization accuracy), through the device and into an OSA.

\section{RESULTS}

Two samples were investigated and produced the best polarisation responses; the first experienced a $3 \mathrm{~dB}$ transmission loss (SLPG-1) or loss of power, whilst the second was deeper and demonstrated a 12dB transmission loss (SLPG-2). Looking at the $3 \mathrm{~dB}$ loss micro-slot long period grating ( $\mu \mathrm{SLPG}$ ), the recorded microscope images are shown in Fig. 1. The images show, from the optical fibre surface to the base of the ablated slot the depth is approximately $7 \mu \mathrm{m}$. Whereas Fig. 1(c) shows that at the widest part of the ablated slot the width is approximately $4.4 \mu \mathrm{m}$.
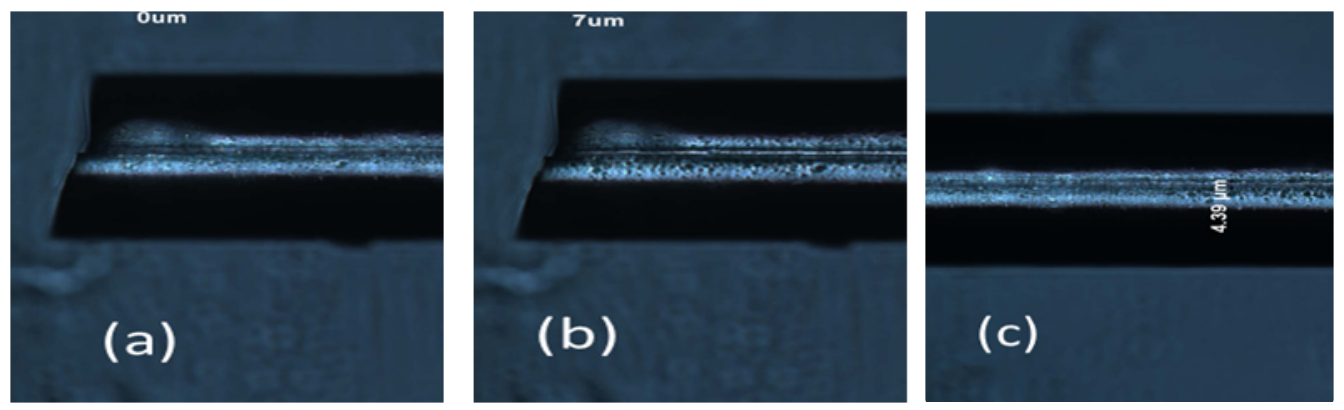

Fig. 1 The ablated groove in SLPG-1 at (a) the surface of the optical fibre, (b) the bottom of the slot and (c) the width of the slot

Plotting the transmission spectra before and after the cladding ablation (Fig. 2) identifies the differences created in the ablation process. The ablation changes the modal structure and distribution; this in turn affects the coupling co-efficient for each mode. This is obvious from a few distinct differences, the attenuation peak located at $1472 \mathrm{~nm}$ (before ablation) has experienced a red wavelength shift, also it's coupling strength has decreased and finally a second attenuation peak is now evident.
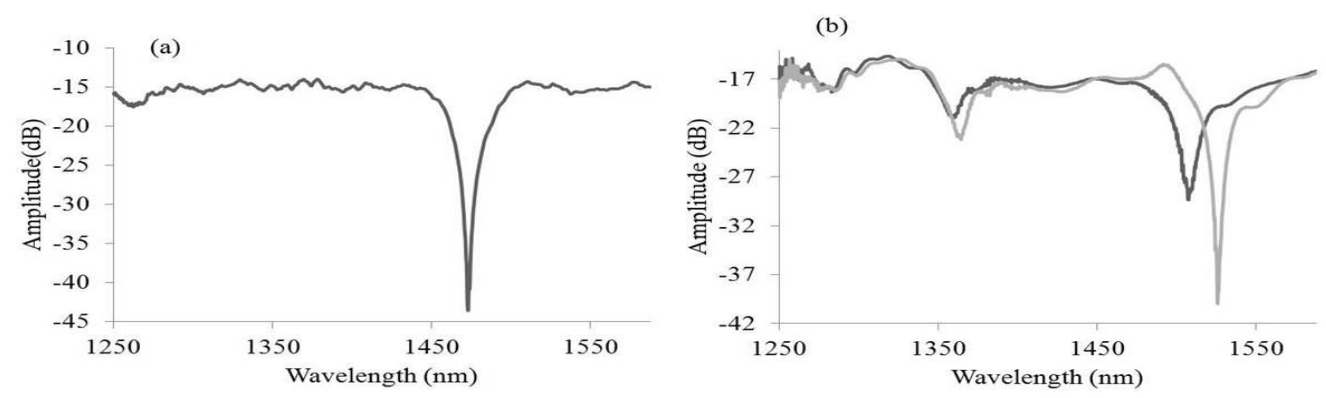

Fig. 2 The transmission spectra for SLPG-1 (a) before the ablation and (b) after the ablation showing the two polarisation peaks for polarisation states $90^{\circ}$ apart 
The important difference to note in Fig. 2(b), is that the attenuation peaks are now displaying a birefringence where none was seen before. We focus on the separated peaks at approximately $1530 \mathrm{~nm}$ wavelength, since they are the strongest. The polarisation response for this peak was evaluated by changing the input polarisation of light from $0^{\circ}$ to $180^{\circ}$ in $10^{\circ}$ increments and calculating the corresponding amplitude change and wavelength shift and this is shown in Fig. 3 . The amplitude of the attenuation peak shrinks and grows, as one would expect with the decrease in strength of one polarisation moving to a growth in strength as the other polarisation begins to dominate. The wavelength polarisation shows a connected response with fixed wavelength position until the input polarisation angle changes significantly, enough for the other polarisation state of the device to dominate. The maximum wavelength and amplitude variation SLPG-1 was calculated to be approximately $13.4 \pm 0.1 \mathrm{~nm}$ and $12 \mathrm{~dB}$, respectively.
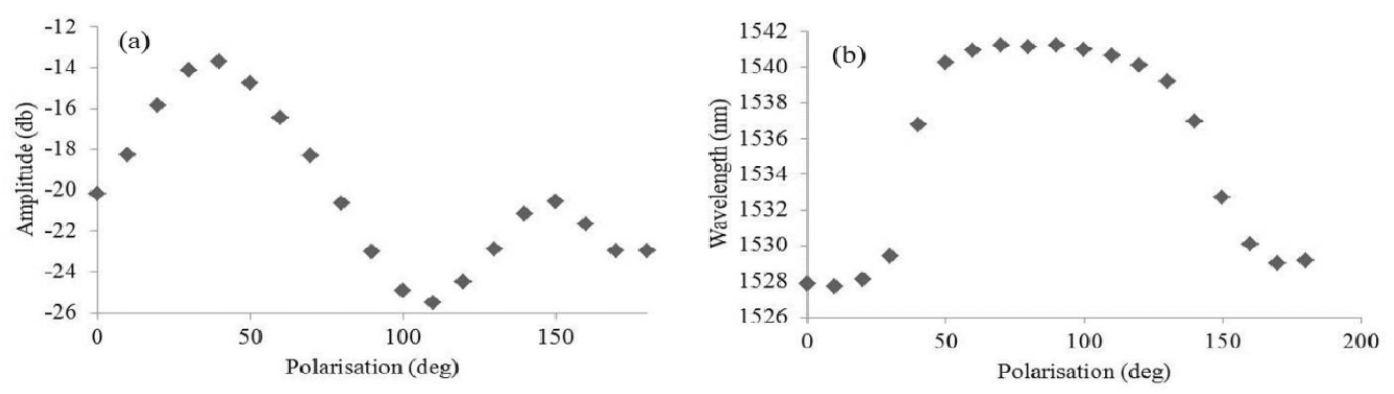

Fig. 3 The polarisation response for the peak at approximately $1530 \mathrm{~nm}$ for (a) the amplitude and (b) the wavelength

Examining SLPG-2 with the microscope produced the images shown in Fig. 4. Two differences are apparent when comparing SLPG-1 to 2. The ablated groove in the optical fibre cladding is now deeper and wider, which is to be expected since SLPG-2 had a far greater transmission loss of 12dB.
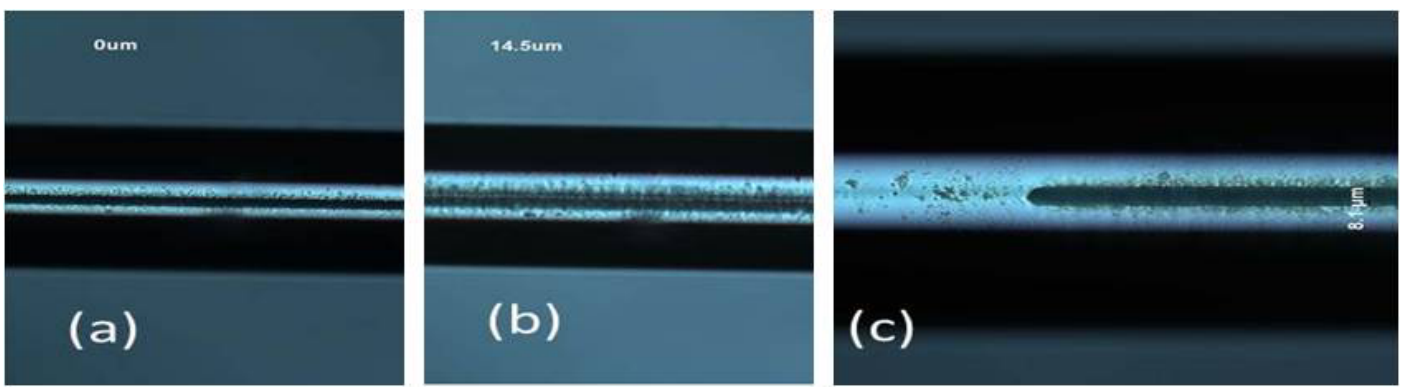

Fig. 4 The ablated groove in SLPG-2 at (a) the surface of the optical fibre, (b) the bottom of the slot and (c) the width of the slot

Examining the spectra before and after the cladding ablation is shown in Fig. 5. The ablation process has again caused a splitting of the attenuation peak associated at $90^{\circ}$ to each other in terms of input polarisation of light. The deeper and wider groove has produced a larger birefringence, greater than that seen in grating SLPG-1, with the attenuation peaks located at approximately $1455 \mathrm{~nm}$ and $1495 \mathrm{~nm}$ (for the strongest attenuation peak). Continuing our examination, the strongest attenuation peak, the polarisation response was evaluated by changing the input polarisation of light from $0^{\circ}$ to $180^{\circ}$ in $10^{\circ}$ increments and calculating the corresponding amplitude change and wavelength shift and this is shown in Fig. 6. The amplitude shrinks and grows as one would expect with birefringent attenuation peaks. The wavelength shift is interesting, as the wavelength separation is so great between the two attenuation peaks that when they shrink and grow, as the polarisation changes, they do not affect each other. This gives rise to distinct wavelength regions of operation and means the device can be highly selective. The measured maximum amplitude variation due changing the input polarisation of light is $10 \mathrm{~dB}$ and the wavelength birefringence was calculated at $39 \pm 0.1 \mathrm{~nm}$. This suggests that with increasing cladding ablation depths comes increased wavelength birefringence, as anticipated. 

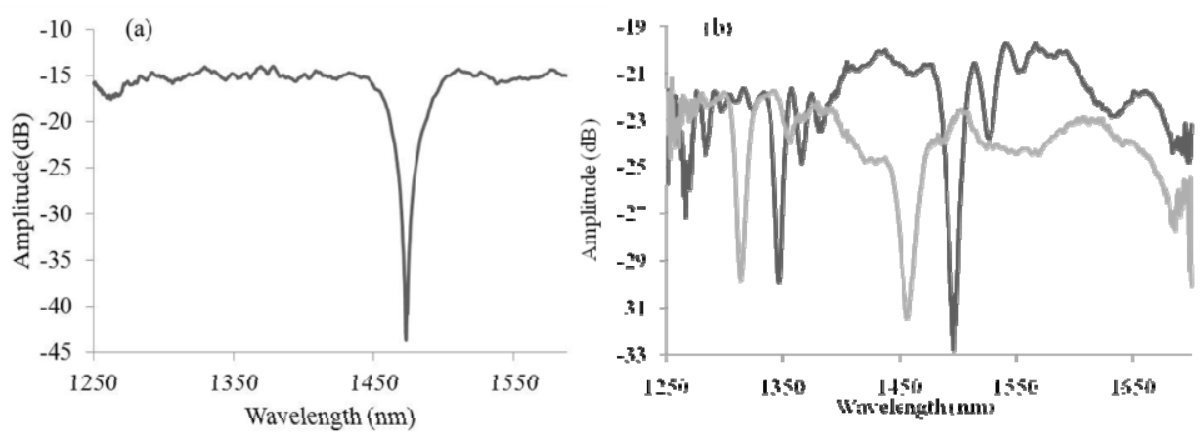

Fig. 5 The transmission spectra for SLPG-2 (a) before the ablation and (b) after the ablation showing the two polarisation peaks for polarisation states $90^{\circ}$ apart

(a)

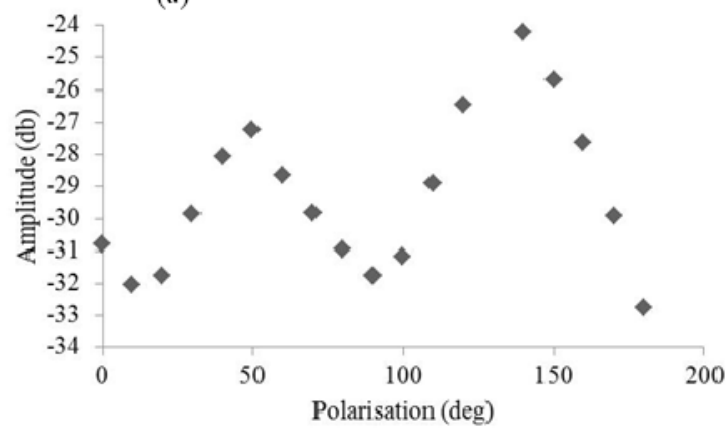

(b)

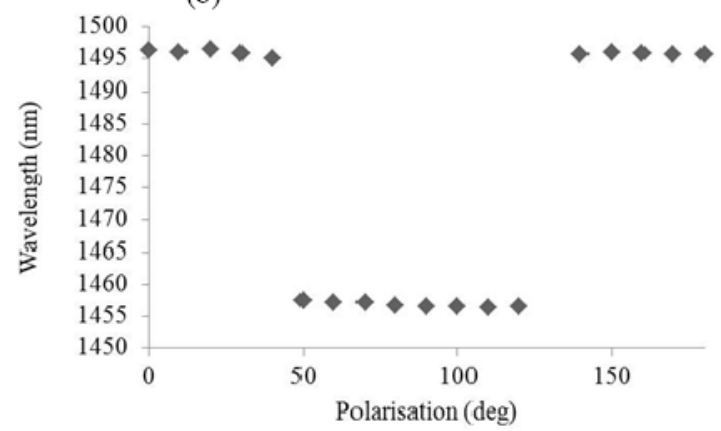

Fig. 6 The polarisation response for the peak at approximately $1496 \mathrm{~nm}$ for (a) the amplitude and (b) the wavelength

\section{CONCLUSION}

Through a simple two step process, of first inscribing a UV gratings in SMF followed by a femtosecond cladding groove ablation, a in line fibre polariser can be produced. A trend is suggested, with increasing ablation groove size comes increased wavelength birefringence. This combined with the UV LPG ability to produce attenuation peaks at almost any wavelength, results in, a polariser that has both wavelength location and birefringence selectivity for any application. The work produced here measured a maximum birefringence of $39 \pm 0.1 \mathrm{~nm}$.

\section{ACKNOWLEDGEMENT}

Edward Davies would like to thank the EPSRC for grant which made his PhD study and this work possible

\section{REFERENCES}

[1] Johnstone W., Stewart G., Hart T., and Culshaw B. "Surface plasmon polaritons in thin metal films and their role in fiber optic polarizing devices", Journal of Lightwave Technology, 8 (4), 538-544 (1990)

[2] Bergh R. A., Lefevre H. C., and Shaw H. J. “Single-mode fibre-optic polariser” Optics Letters, 5 (11), 479-481(1980)

[3] Zhou K., Simpson G., Chen X., Zhang L., and Bennion I. "High extinction ratio in-fiber polarizers based on 45 tilted fiber Bragg Gratings" Optics Letters, 30 (11), 1285-1297 (2005) 